\title{
COESISTENZA/CONVIVENZA
}

\section{TRA EBREI E CRISTIANI ISPANICI}

\author{
DAVID ROMANO
}

Universitat de Barcelona

\section{ÍNDICE}

0. Introduzione

0.1 Impostazione del problema

0.2 Definizione dei termini coesistenza/convivenza

0.3 Definizione di altri termini: minoranza/ tolleranza/ emarginazione

0.4 La terna: filosofia, teoria, realtd

0.5 Atteggiamenti antitetici: $i$ punti di vista cristiano ed ebreo

0.6 Aspetti da studiare

1. La filosofia

2. La teoria

3. La realtà

3.0 Prolegomeni

3.1 Le decisioni del IV Lateranense

3.1.1 Limitazioni delle usure eccessive

3.1.2 Divieti di occupare cariche pubbliche

3.1.3 Obbligo di indossare vestiti e segni distintivi

3.1.4 Separazione di habitat

3.2 Diversitd di costumi: due mogli contemporaneamente

3.3 La rottura della coesistenza e della tolleranza

3.3.0 Linee di forza

3.3.1 I principali fatti cronologicamente disposti

3.3.2 Le rotture mentali

3.3.2.1 Le prediche cristiane

3.3.2.2 Gli addottrinamenti cristiani

4. Conclusione 


\section{INTRODUZIONE '}

\subsection{Impostazione del problema}

Penso che nessuno dubita che cristiani ed ebrei ispanici abitassero nelle stesse città; invece, però, le discrepanze sono notorie per quanto riguarda la maniera di abitare in codeste città.

Penso che sia necessario frenare e addirittura eliminare la sopravvivenza e diffusione di un errore che è essenziale in sè stesso. Si parla troppo spesso di convivenza di ebrei con musulmani e cristiani. A dire il vero, tanto in territorio musulmano come in territorio cristiano, quel che ci fu fu una mera coesistenza, tanto pacifica e perfino cordiale come si voglia, ma coesistenza in fin dei conti. In certe occasioni possibilmente ci furono anche buoni rapporti, anche certa convivenza, per esempio, tra le coppie di cotraduttori delle quali ho parlato con qualche frequenza ${ }^{2}$. Altresì, restano notizie di rapporti sessuali tra persone di religione diversa, mentre che non c'è nessuna prova irrefutabile, manca assolutamente qualsiasi attestazione, di matrimoni misti: non ce ne furono mai, non ci potevano essere. Similemente, conosciamo abbondanti documenti (acquisti, vendite, prestiti, ecc.) riguardanti rapporti economici tra ebrei e

' Ecco il testo della relazione presentata a Genova (25-28.5.1992) al congresso «1492-1992 Le vie difficili della convivenza. Gli ebrei sul filo della storia». Rispondendo a una mia domanda, l'editrice Marietti mi fa sapere (17.1.1995) che «la pubblicazione degli atti del convegno... non rientra nei nostri programmi editoriali». Per ciò decido di farla pubblica in Sefarad. Questo studio si è giovato parzialmente dell'aiuto del progetto $\mathrm{n}^{2}$ PB90-0449-C02-01 della Dirección General de Investigación Científica y Técnica (DGICYT), del Ministerio de Educación y Ciencia spagnolo. Molte delle cose che dirò provengono da relazioni presentate in congressi, soprattutto dell'anno 1991. Ciò mi permette di risparmiare i riferimenti generici e di limitarmi alla bibliografia specifica. Sul perché dell'uso del termine 'ispanico' invece di 'spagnolo', ho parlato di recente nella relazione D. ROMANO, "Judíos frente a cristianos en la Edad Media hispánica", relazione al congresso "1492 - A Turning Point", organizzato dall'Università di Tel-Aviv (Madrid 11-13.5.1992).

2 D. Romano, "Le opere scientifiche di Alfonso X e l'intervento degli ebrei", in Oriente e Occidente nel Medioevo: Filosofia e Scienze. Convegno Internazionale 9-15 aprile 1969, Roma 1971, 677- 711 [ristampato in D. RomANo, De historia judia hispánica, Barcelona 1991, 147-183]; e IDEM, "La transmission des sciences arabes par les Juifs en Languedoc", in Juifs et judaisme de Languedoc, Toulouse 1977, 363-386 [ristampato in D. Romano, De historia, 209-232]; e poi IDEM, “El papel judio en la transmisión de la cultura", Hispania Sacra XL [Congreso Internacional de Historia eclesiástica comparada] (1988) 955-978 [ristampato in D. Romano, De historia, 431454]. 
cristiani, magari anche associati. I contatti si riducevano soltanto alla sfera sociale, alla sfera economica, alla sfera della coesistenza più $o$ meno pacifica.

Coesistenza e convivenza sono due termini indubbiamente non sinonimici benché talvolta, spesso, troppo spesso, vengano intercambiati senza nessuna esitazione. È questo un soggetto che tengo a cuore e ne ho parlato sovente; ma ne ho parlato di sfuggita ${ }^{3}$, tranne in due occasioni ${ }^{4}$ : una generica, in una lezione di un corso di aggiornamento universitario; un'altra in un congresso, pur se allora pensavo soprattutto al periodo tra 1391 e 1492, due date che ora non vorrei spiegare, tranne che sia necessario nel dibattito.

Questi due termini sono strettamente legati ad altri due termini che vengono presupposti: tolleranza ed emarginazione, che ricorreranno spesso in questa mia relazione. Ed anche altri due: minoranza ed inferiorità degli ebrei, di fronte a maggioranza e superiorità dei cristiani.

Bisognerebbe definire i due termini principali (ed anche gli altri ambi di parole). Non è stato fatto, e non è facile a farsi, soprattutto in italiano. Per ragioni non linguistiche, cioè, per ragioni politiche del passato è da rilevare la mancanza di un dizionario ufficiale della lingua italiana s. Dato che questo non esiste, in via di ipotesi in questa relazione utilizzerò ambedue parole in senso ristretto, senza conceder ad esse un valore assoluto.

${ }^{3}$ D. Romano, “Característiques dels jueus en relació amb els cristians en els estats hispànics", in Jornades d'Història dels jueus a Catalunya, Girona abril 1987, Girona 1990, 9-27 e 29-42 [ristampato in D. ROMANO, De historia, Barcelona 1991, 475-493]; ÍDEM, "Rasgos de la minoría judía en la Corona de Aragón», in Xudeos e Conversos na Historia. II: Sociedade e Inquisición (Actas do Congreso Internacional Ribadavia 14-17 de outubro de 1991), Santiago de Compostela 1994, 221-246; IDEM, «Perspectivas de la historia judía de la Corona de Aragón" Espacio. Tiempo y Forma, serie III, 6 (1993) 301-334, relazione al congresso "En torno a Sefarad", Toledo 16-19.12.1991; ecc.

4 Vedasi D. Romano, "Los judíos hispánicos entre tolerancia y marginación", conferenza nel corso (Universidad de Alcalá de Henares) su "Tolerancia y marginación en la Edad Media" (Sigüenza 1.7.1991) poi esposta nella relazione "Judíos hispánicos: coexistencia, tolerancia, marginación (1391-1492) (De los ataques a la expulsión)", relazione alle III Jornadas hispano-portuguesas de Historia Medieval "La Península Ibérica en la Era de los descubrimientos, 1391-1492», sezione "El final de la convivencia: Judíos y conversos en la Península Ibérica al final de la Edad Media», Sevilla 25- 29.11.1991, tuttora inedita.

${ }^{5}$ La mancanza di un dizionario è dovuta all'inesistenza di una Accademia della Lingua, dopo l'efimera vita di quella creata a Roma, e non sostituita né dai Lincei né dalla Crusca. 
Ad ogni modo, questi non sono concetti odierni. Se vogliamo avvicinarci alla realtà medioevale, alle realtà medioevali in plurale, bisogna tener presente che di fatto la religione costituiva un motivo di separazione, una vera barriera, che niente potè distruggere, nella Penisola Iberica nemmeno l'espulsione. Agli ebrei era tollerato di osservare la loro religione, e veramente pagavano buon prezzo per questa tolleranza; ma era vietato svolgere la minima attività di proselitismo. (Bisogna ricordare che la conversione all'ebraismo è molto difficile). Insisto: c'era coesistenza ma non convivenza.

Io direi che è quasi un assurdo parlar di convivenza quando una delle parti cerca senza indugi la conversione dell'altra (sto pensando, fra l'altro, agli addottrinamenti di Barcellona e di Tortosa). La reciproca, cioè, che gli ebrei mirassero ad una giudaizzazione dei cristiani, è o sarebbe stata certa? La risposta è mancante, tranne in via ipotetica: i ceti dominanti erano cristiani, mai furono ebrei. Quando, però, le persone in situazione di inferiorità si sono convertite, allora caso mai si può parlare di convivenza ma soltanto tra persone e non tra gruppi diversi, sostanzialmente diversi.

\subsection{Definizione dei termini coesistenza/convivenza}

Dal punto di vista teorico, convivere è un verbo valido soltanto tra due parti uguali ma non differenti. C'è una premessa essenziale: che ci sia libertà, anche se soltanto di ordine teorico. Invece, coesistere non richiede necessariamente che le due parti si trovino in posizioni d'ugualità, ed infatti talvolta si parla di coesistenza pacifica: per definizione la convivenza suol essere pacifica. Credo che sarebbe degno di sforzo aproffondire in quello che chiamo precisazioni terminologiche ${ }^{6}$. Bisogna meditare sull'apparente, ma falsa, sinonimia fra convivenza e coesistenza, e specie sull'uso spesso abusivo della parola convivenza, alla quale forse bisognerebbe offrire libera via d'uscita: nella Penisola Iberica, di sicuro in qualsiasi altra zona del mondo, fra ebrei e cristiani mai non ci fu convivenza, bensì nel migliore dei casi semplicemente coesistenza ed infatti molti parlano di distanza sociale, che non è altro che una scorciatoia per evitare l'uso della parola coesistenza. Per esempio, in francese si parla di

${ }^{6} \mathrm{Si}$ veda soprattutto il $\S 0$. della mia relazione «Judíos frente a cristianos en la Edad Media hispánica" (citata nella nota 1). 
cohabitation, parola che in italiano (ed anche in spagnolo) ha due sensi: uno è quello di coesistenza ed un altro quello di contubernio.

E poi bisogna distinguere convivenza/coesistenza tra persone e tra gruppi, bisogna stabilire con proprietà l'uso dei termini somiglianza/ugualità ed anche differenza/disugualità.

Ho l'impressione che sarebbe interessante far la storia dei termini. Non starò ora a farla. Badate, però, che i dizionari italiani, sia storici che di autorità (Battaglia, per esempio), attestano la presenza di questi sostantivi non prima della fine del 700 e soprattutto dall'epoca romantica in poi, sebbene $\mathrm{i}$ verbi sono già stati rilevati nel medioevo.

In questa relazione mi occuperò di casi concreti su quest'argomento. Ma ora tengo a sottolineare un fatto sul quale non mi dilungherò: tutti quanti siete consci che la differenza di religione ha un peso straordinariamente forte nei rapporti individuali e sociali, peso che era ancor più grande nel medioevo. Numerosi dati ed idee prendono l'avvio dalla differenza di religione.

\subsection{Definizione di altri termini: minoranza/tolleranza/emarginazione}

Non c'è il menomo dubbio che nelle nazioni ispaniche gli ebrei costituivano una minoranza. Ma, però, fino ad ora non si è potuto -sarà possibile farlo in futuro? - esprimere in cifre il valore del termine minoranza, in nessuno degli aspetti principali che possono venir considerati: 1) il calcolo delle cifre assolute, e 2) la percentuale di ognuno dei gruppi.

Anticipando alcuni dei risultati provvisori di un lavoro in corso ?, limitato alla Corona d'Aragona, indicherò che per quanto riguarda le cifre assolute di popolazione il cammino soltanto è stato leggeramente dissodato; non possiamo pensare di essere vicini alla realtà tranne che per località di scarsa popolazione ebrea, sempre che si accetti il coeficente famigliare 4,5 (ó 4,12). Malgrado la riduzione demografica, anche se il potenziale ancora non è stato calcolato, il numero di ebrei che continuarono ad esserlo dopo i moti del 1391 fu relativamente forte; ma la coesistenza e la tolleranza dei cristiani

\footnotetext{
${ }^{7}$ Con il titolo «Demografia judía de la Corona de Aragón: planteo sistemático para determinar cifras de población", detti una conferenza (Madrid 27.8.1990) nella riunione "Spanish Jewry and its Dispersion: Its Influence on the History of Spain and on Jewish History", annessa al 17th International Congress of Historical Sciences.
} 
sprofondarono poco a poco; come contrappeso, aumentò l'emarginazione.

Una minoranza rarissimamente è amata. Il fatto stesso di essere una minoranza, tradotto in parole povere vuol dire inferiorità.

Pensando come lo avrebbe fatto un cristiano medievale sembra chiara la superiorità della religione cristiana sull'ebraica e di conseguenza quella del cristiano sull'ebreo $o$, detto in altro modo e più apertamente, l'inferiorità dell'ebreo. Ma forse ci troviamo immersi nel vecchio dilemma di sapere cosa fu prima: l'uovo o la gallina?

Poi arriva il rispetto, ossia, rispetto dopo aver messo l'ebreo in situazione di inferiorità. Questa situazione è manifesta in un mucchio di fatti a carattere sociale, o semplicemente materiale, ancora mancanti di un coerente studio sistematico.

L'ebreo può osservare privatamente la sua religione ma sempre dopo aver accettato la situazione di inferiorità legale e sociale, e forse altre ancora.

Dal punto di vista del potere, almeno di quello delle nazioni ispaniche, la situazione di inferiorità significa emarginazione e tolleranza, cioè tolleranza dentro emarginazione. Insisto sul fatto che coesistenza non equivale a convivenza, giacché esistevano allora ed esistono tuttora una serie di luoghi comuni antiebrei che bisognerebbe esiliare definitivamente.

In termini generali, i sovrani riconoscono le esclusioni decretate dai rabbini mediante alatma, herem o nitduy. Riconoscono anche la validità dei documenti ebrei ed, in genere, gli atti validati da notai ebrei, la giurisdizione (con talune limitazioni) dei giudici ebrei quando i contendenti sono ebrei, cioè, tutto quanto può venir qualificato come tolleranza dentro dell'emarginazione, come sarebbe il caso della legittimità di due mogli contemporaneamente.

Vi sono, comunque, diversi gradi di tolleranza ed emarginazione. E ben evidente che gli ebrei vengono tollerati in contraccambio d'emarginazione. Ed aggiungerò: con la contropartita che paghino, anche se è considerato brutto imputare questo (ricatto?) a signori cristiani di nazioni cristiane; ma è la verità, chiamata allora e/o ora in altre forme o maniere, dicendo, per esempio, contribuiscano, aiutino o appoggino economicamente.

E subito scaturisce una domanda: cosa è anteriore, il rispetto o la situazione di inferiorità? In altre parole: la tolleranza o l'emarginazione? 


\subsection{La terna: filosofia, teoria, realtà}

Penso che molte questioni dovrebbero venir abbordate da parecchi punti di vista. Inoltre, bisognerebbe fare delle analisi tomografiche a livelli, cioè, non parlare di tutti i cristiani complessivamente, bensì cercare di differenziare tra quello che pensava e/o faceva il sovrano, il clero, la borghesia, il popolo, e questo non in astratto ma piuttosto in concreto, ossia, i sovrani, i cleri, le borghesie, $\mathrm{i}$ popoli.

L'idea della possibile validità di una terna nell'analisi dei fatti storici, mi venne in mente quasi un ventennio fa, una terna che potrebbe esprimersi in tre parole: filosofia, teoria, realtà. L'impostazione più sistematica la feci per la prima volta in un saggio su Alfonso X di Castiglia (1252-1284) e gli ebrei ${ }^{8}$, saggio che ora non vorrei né ripetere né esporre sommariamente. Ma, però, penso che è doveroso sottolineare il fatto che non è possibile separare decisamente filosofia da teoria, né teoria da realtà. I fatti mai sono asettici e non lo sono nemmeno i documenti. Spesso questi elementi presi due a due mischiano concetti, e spesso li mischiano volutamente.

Ad ogni modo, in linea di massima pare evidente che nei due primi elementi, cioè, filosofia e teoria sono presenti la tolleranza e l'emarginazione; invece, nel terzo elemento, la realtà, di solito soltanto è documentata l'emarginazione mentre la tolleranza forse sarà mentalmente presente ma non è manifesta. Ciò spiega che ora mi occupi piuttosto della realtà che degli altri due aspetti.

\subsection{Atteggiamenti antitetici: $i$ punti di vista cristiano ed ebreo}

Nella Penisola Iberica i rapporti cristiani-ebrei - tralascio ora i musulmani- in linea di massima dovrebbero venir analizzati da un duplice punto di vista, secondo il proverbio spagnolo: «todo es según el color del cristal con que se mira". Intanto, in questo momento quello che domina è il punto di vista cristiano, cristiano del Medioevo ispanico, l'unico che ora considererò. Sarebbe ben più che interessante conoscere cosa pensavano gli ebrei dei cristiani rispetto a tanti problemi, specie rispetto a coesistenza e convivenza,

${ }^{8}$ D. Romano, "Alfonso $\mathrm{x}$ y los judíos. Problemática y propuestas de trabajo", Anuario de Estudios Medievales 15 [ = Estudios dedicados a la memoria de D. Claudio Sánchez-Albornoz] (1985) 153-162 [ristampato in D. Romano, De historia, 375-384]. 
e a tolleranza ed emarginazione. È un soggetto che non è stato ancora studiato. Ed una questione interessante sarebbe domandarsi: è possibile studiarlo? Bisognerebbe leggere oltre a molte fonti ebraiche teoriche, i Responsa dei rabbini ispanici, che possono orientarci sull'argomento.

Il punto di vista ebreo può essere ed è senz'altro interessante; ma però bisogna rilevare che gli ebrei non sono i dirigenti e dunque non hanno la possibilità di tradurre le loro idee in realtà.

Questo mi porta a ricordare, pur molto brevemente, la questione delle fonti, tema fondamentale che mi attira da moltissimo tempo". La buona storia è basata su materiali coetanei, ma l'interpretazione può farsi in qualsiasi epoca. A mio avviso l'importante sono le notizie documentarie e non quelle provenienti da testi, sempre rimaneggiati in maggior o minor misura. Ma una buona storia ispanoebrea deve fondarsi su fonti ebraiche e fonti cristiane e bisogna tener conto di ambedue le possibilità euristiche.

Mettiamo per caso il prestito. Secondo il Talmud un ebreo non può prestare ad un altro ebreo; secondo il diritto canonico un cristiano non può prestare ad un altro cristiano. Ma non c’è nessun divieto, anzi, io direi che quasi viene incoraggiato il prestito a persone di religione diversa. A dire il vero, sono stati studiati, troppo studiati, i prestiti degli ebrei ai cristiani; viceversa, il prestito di cristiani ad ebrei ha destato scarso interesse. Comunque, ripeto che gli ebrei non hanno la possibilità di trasformare filosofia e teoria in realtà.

D'altronde, allo stato attuale della ricerca oggidì soltanto è possibile gettare lo sguardo dalla prospettiva cristiana.

Malgrado l'atteggiamento in termini generali tollerante, quando si mirava a convertire gli ebrei al cristianesimo la rottura della tolleranza e della coesistenza si esprimeva di diversi modi. La Penisola Iberica fu scenario di attacchi a carattere religioso contro gli ebrei: attacchi orali e scritti, e poi ad attacchi di fatto, sia regolari (p. es. Settimana Santa) sia non regolari: pastoureaux, 1348, 1391, ed espulsione.

\footnotetext{
${ }^{9}$ Seguo da vicino quanto dissi nel $\S 2.1$ nella mia relazione «Perspectivas de la historia judía de la Corona de Aragón" (citata nella nota 3).
} 


\subsection{Aspetti da studiare}

La prima base che stabilisce l'impostazione della situazione dell'ebreo medievale fu delineata dalle disposizioni del IV Concilio Laterano (1215), disposizioni che rivelano un atteggiamento di emarginazione + tolleranza, apertamente manifesto in quattro questioni: 1) le usure eccessive - sottolineo queste eccessive-, 2) il divieto di occupare cariche pubbliche, 3) il distinguersi dall'abbigliamento, e 4) la separazione fisica. A queste quattro questioni si deve aggiungere un'altra implicita e che testualmente non è chiara che fosse rivolta agli ebrei: sto pensando alla medicina.

Sono punti la cui analisi contribuisce a dimostrare la mancanza di convivenza, mancanza già nota sin dall'inizio del secolo IV, momento della riunione del concilio di Iliberis, che offre la prima testimonianza sicura e datata degli ebrei ispanici ${ }^{10}$.

Dichiaro, però, che non mi impegnerò a tracciare una visione cronologica, che probabilmente in quest'occasione non compierebbe i fini proposti. Eppure, ora vorrei esporre alcuni aspetti da tenere in mente come retrofondo di quello che dirò poi. La situazione degli ebrei complessivamente mai fu buona: nel migliore dei casi era tollerabile perché era il risultato di una tolleranza.

\section{LA FILOSOFIA}

Sarebbe ben necessario compilare un elenco particolareggiato delle notizie sulla posizione mentale dei cristiani medievali riguardo agli ebrei ". E questo lasciando in disparte l'uso e diffusione dei testi strettamente dottrinali, e soffermandosi anche sui dati che rivelano la non esistenza di convivenza. Non starò ora a farlo. È una ricerca che richiede molto tempo. Invece vorrei più modestamente accennare ad alcuni testi a mio avviso significativi in se e per la repercussione che ebbero.

${ }^{10}$ D. Romano, "Judios hispánicos en los siglos IV-IX", relazione al III Congreso de Estudios Medievales (León 23-27.9.1991) e pubblicata nel volume De la Antigüedad al Medioevo (siglos IV al VIII), Madrid 1993, 251-265 e 276-278.

$11 \mathrm{Si}$ possono leggere alcuni suggerimenti in D. ROMANO, "Penseurs chrétiens catalans vis-à-vis des juifs", relazione presentata al congresso "Juden und Judentum in der Sicht der christlichen Denker im Mittelalter" (Wolfenbuttel 18-20.10.1993), tuttora inedita. 
La prima cosa sarebbe valutare tutto quanto si potrebbe desumere da un'accurata lettura dei testi del IV Lateranense dove, per esempio, viene opposta la christiana devotio alla iudeorum perfidia, espressione quest'ultima già presente in testi ispanici ben più antichi, visigoti del secolo VI, come sarebbe il VI Concilio toledano, che menziona la “inflexibilis iudaeorum perfidia" ${ }^{2}$.

Se ora ci trasferiamo al Basso Medioevo non credo che sia inutile ricordare le manifestazioni di Alfonso $\mathrm{X}$ di Castiglia che accenna spesso agli ebrei e addirittura definisce

Judíos son una manera de homes que como quier que non creen la fe de nuestro señor Jesucristo, pero los grandes señores de los cristianos siempre sufrieron que viviessen entre ellos ${ }^{13}$.

E se, sempre nel Basso Medioevo, facciamo il salto ad un'altra nazione - la Corona d'Aragona - ed a un altro livello - quello religioso- ci troviamo davanti a quello che dicevano gli addottrinamenti, di Barcellona e di Tortosa, come spiegherò poi (§ 3.3.2.2).

Tutto arriva al parossismo in certi momenti specialmente critici, volutamente critici, per esempio, nella fraseologia dell'editto d'espulsione dei $\operatorname{Re}$ Cattolici, in area teoricamente civile - non $\mathrm{mi}$ trattengo ora sull'origine di questo atteggiamento.

Un documento o testo - non riesco a definire cosa sia - fatto conoscere di recente ${ }^{14}$ e sul cui valore e significato tengo in sospeso il mio giudizio, si trattiene sulla malvagità dell'atteggiamento e delle attività degli ebrei che hanno come scopo indurre i cristiani (nuovi) all'apostasia e all'eresia. Adopera frasi come "diabólica y pérfida inducción e suggestión de los dichos judíos", allude al loro «inquieto y perverso bivir», ed esprime la sentenza "nos fuesse lícito y permeso segund su perfidia y segund los dichos actos tan nefarios y detestables por ellos cometidos ... que por su obstinada infidelidat son incorregibles, punirlos de mayores y más grandes penas", "que pervierten el casto y honesto bivir".

12 J. Tejada y Ramiro, Colección de Cánones y de todos los concilios de la Iglesia de España y América, Madrid 1859. 8).

${ }^{13}$ Copiato nel $\S 2$. di D. Romano, "Alfonso X y los judíos" (citato nella mia nota

14 R. Conde y Delgado de Molina, La expulsión de los judios de la Corona de Aragón. Documentos para su estudio, Zaragoza 1991, documento n 1, pag. 41-44. 
Il ricordo di questi frammenti suscita subito la domanda retorica: è giusto parlare di convivenza?

\section{LA TEORIA}

La teoria di solito viene espressa nelle leggi, che sono facili ad essere riunite e studiate specie quando sono inserite nelle compilazioni ${ }^{15}$.

Infatti, la soluzione teorica derivante dai passi citati è diafana: bisogna prendere delle misure, decidere cosa dovrebbe farsi per evitare qualsiasi sentore di convivenza ed eventualmente per ridurre o addirittura eliminare la coesistenza. Nel 1492 pare che la misura più netta sia l'esilio, eliminare gli ebrei da questa zona geografica, politica se volete, benché non sia scartata la possibilità della coesistenza fuori delle frontiere dei Re Cattolici.

Facciamo ora dietro front e ritorniamo alla fine del '200. Basta gettare uno sguardo sul pensiero di Alfonso $\mathrm{X}$ di Castiglia che allacciando filosofia e teoria svolge una larga attività giuridica che però spesso è contraddetta dalla realtà ${ }^{16}$. E se cambiamo di nazione e passiamo alla Corona d'Aragona, il panorama che ci si offre è ben manifesto. Dei moltissimi esempi teorici che potrebbero ricordarsi, uno dei meno noti procede da certe costituzioni sinodali di Lerida (1325) ${ }^{17}$, che decretano la scomunica delle cristiane che convivessero -sottolineo questa parola - con degli ebrei ${ }^{18}$, ed allo stesso tempo la scomunica dei cristiani presenti alle circoncisioni ed alle nozze ebraiche allo scopo di onorare i protagonisti ebrei ${ }^{19}$.

Di poco posteriore è un altro esempio, sempre della Catalogna.

${ }^{15} \mathrm{Si}$ veda per esempio D. Romano, «Marco jurídico de la minoría judía en la Corona de Castilla de 1214 a 1350. (Síntesis y propuestas de trabajo)", in Actas del II Congreso Internacional: Encuentro de las tres Culturas, Toledo 1985, 261-291 [ristampato in D. Romano, De historia, 341-371].

16 Vedasi D. Romano, "Alfonso X y los judíos" (citato nella mia nota 8).

17 J. Villanueva: Viage literario a las iglesias de España, Madrid 1806-1852, XVII, pag. 243.

18 "Quod mulieres christianae morantes cum judeis sunt excomunicatae, et debent publicare quolibet anno ubi judei morantur".

19 "Quod Christiani non intersint circumcisionibus vel nuptiis judeorum causa honorandi; alias sunt excomunicati». 
Nel 1345 un ebreo della città di Girona venne punito - traduco dall'originale catalano-

perché disse ad un macellaio di Girona che rinegherebbe Dio e tutti i cristiani prima di accettare che suo fratello diventasse cristiano ${ }^{20}$.

E trenta anni più tardi (1377) a Barcellona fu fatta una leva che indica - traduco dall'originale catalano-

Fu deciso che gli ebrei andassero con il sottoveguer e la sua cinquantina per fare le suddette guardie o perquisizioni, perché se fossero messi in un altro luogo della città sarebbe da temere che venissero dannificati dai cristiani della suddetta città ${ }^{21}$.

Guardate che non ci troviamo davanti ad un testo religioso o un testo programmatico, ma semplicemente davanti ad un censimento militare nel quale viene messa in rilievo la neccessità di postare gli ebrei in un altro posto, cioè, di metterli in luogo diverso dai cristiani, per il pericolo che la vicinanza avrebbe potuto significare.

I due ultimi esempi palesano, senza dubbi ne esitazioni, l'atteggiamento di ognuna delle parti: non c'è propriamente convivenza ma semplicemente una coesistenza, giacché la religione marca una differenza assoluta tra cristiani ed ebrei.

E vi sono tantissimi altri casi. Nel ' 400 i rapporti tra ebrei e cristiani nuovi (cioè, convertiti) persistono sulla stessa scia, appaiono uguali pur essendoci circostanze nuove ${ }^{22}$.

L'effettività di queste misure concrete è evidente. Ma in termini generici pare che la vita racconti una tutt'altra storia, offra prospettive diverse, cioè, la realtà di cui parlerò subito.

${ }^{20}$ "Pero ço com dix a un carnicer de Girona que abans renegarie ell Deu e tots quants cristians eren que son frare no.s farie christia".

21 "Fo acordat que los juheus anassen ab lo dit sotsvaguer e ab la dita cinquantena ensemps per fer les demunt dites guaytes o serques, e aço per tal com si en altre loch de la ciutat foren posats fore dubte que no fossen damnificats per los crestians de la dita ciutat", F. MARSÁ, Onomástica barcelonesa del siglo XIV, Barcelona 1977, pag. 173.

${ }^{22}$ Si veda specialmente il primo paragrafo, e la nota 1, dell'articolo di E. MARÍN Padilla, «Relación judeoconversa durante la segunda mitad del siglo XV en Aragón: nacimientos, hadas, circuncisiones", Sefarad XLI (1981) 273-300, 273. 


\section{LA REALTA்}

\subsection{Prolegomeni}

La teoria può tacere, e tace, su moltissimi punti; ma quando si pone una data situazione e si risolve, allora la teoria si lascia intravedere come la punta di un iceberg. Comunque, penso che la convivenza era nulla o straordinariamente scarsa, situazione che diventa chiara in una serie di casi: si mira quasi sempre ad evitare la convivenza e, dunque, la possibile superiorità degli ebrei anche se talvolta si accettino, velis nolis, situazioni di fatto, non volute.

Senza aver l'intenzione di approfondire quest'argomento, premetto un'idea di indole generica sottogiacente. Le occupazioni preferite dagli ebrei erano quelle che permettevano di ricominciare di nuovo nell'eventualità di allontanamento, emarginazione, esilio, ossia, tutto quanto si fondava su "materia prima transportabile», cioè, l'artigianato, la medicina, le finanze. Di conseguenza, dominava un evidente distacco dall'agricoltura.

Una sintesi riassuntiva delle regolazioni medioevali sugli ebrei e specie della loro applicazione reale, mette in rilievo che tra cristiani ed ebrei c'era una separazione giuridica (gli ebrei non sono presenti nelle Cortes, pagano separatamente, non possono occupare cariche pubbliche, non possono frequentare le Università) che magari poteva arrivare ad essere una discriminazione giudiziaria patente nella testimonianza (due testimoni, talvolta, in circostanze concrete, uno di ogni gruppo) ed anche nell'esistenza di varie vie giudiziarie o forse soltanto una duplice giurisdizione: se uno dei litiganti è cristiano, naturalmente è obbligatorio che i giudici siano cristiani; se tutti i due litiganti non sono cristiani, per esempio, sono ebrei, si può scegliere: tribunale rabbinico o tribunale cristiano. È questo un argomento che chiama a gran voce volontari pur se richiede grandi strapazzi.

Inoltre, penso che bisogna ricordare che gli ebrei sono assenti nell'habitat rurale ${ }^{23}$.

${ }^{23}$ D. Romano, «Judíos hispánicos y mundo rural», Sefarad LI (1991) 353-367. 


\subsection{Le decisioni del IV Lateranense}

Riprendiamo le decisioni del IV Lateranense, cioè, le quattro questioni concernenti: l'usura, le cariche pubbliche, l'abbigliamento, la separazione fisica. Non c'è dubbio che questi quattro aspetti non sono i soli, ma bisogna pur limitarsi. La lettura di codeste decisioni e soprattutto la loro applicazione pratica rivela palesemente un'emarginazione, quindi una non convivenza.

\subsubsection{Limitazioni delle usure eccessive}

La prima decisione riguarda l'usura, che nel vocabolario di quel tempo soltanto significava interesse. Infatti, un canone spiega che «judei a christianis graves immoderatasve usuras extorserint». Penso che bisogna badare a questi aggettivi graves e immoderatas. Non so se si pensava che gli alti interessi dei crediti fossero inerenti agli ebrei; però sta di fatto che non si fa parola delle attività creditizie dei cristiani pur se è palese che queste esistevano e che le esercitavano perfino membri della Chiesa ${ }^{24}$.

Dato, ma non concesso, che, come corollario di intoppi giuridicomorali, praticamente venisse conferito, per dirla in forma elegante, alle minoranze non cristiane il monopolio delle attività pre-bancarie, se si pensa che l'intenzione era arrivare all'emarginazione degli ebrei, il tema entra nel terreno delle motivazioni religiose o pseudoreligiose.

L'esempio più evidente, a mio parere perché è il più documentato, è il prestito ${ }^{25}$, netto tentativo di emarginazione nonostante che vi siano testimonianze che rivelano che anche $\mathrm{i}$ cristiani prestavano ${ }^{26}$.

${ }^{24}$ Per esempio, nel 1284 i templari di Aragona e Catalogna prestarono all'infante Alfonso (il futuro re Alfonso il Liberale) 10.000 soldi giacchesi "sub usuris" (ACA., Reg. 71, fol. 15). Questo infante Alfonso potrebbe essere «lo giovanotto che dietro a lui siede" del Purgatorio VII, 116.

${ }^{25}$ Il primo avvicinamento al tema lo feci nell'articolo D. ROMANO, «Prestadores judíos en los estados hispánicos medievales", Estudios Mirandeses VIII (1988) 117-126 [ristampato in D. Romano, De historia, 421-430]. Ora lo sto abbordando in una ricerca in corso, $\mathrm{i}$ cui primi risultati furono esposti in una conferenza "El crédito judío", nel ciclo su Dinero, banca y crédito en la Edad Media della XVIII Semana Internacional de Estudios Medievales (Barcelona 26.6.1990).

${ }^{26} \mathrm{Ne}$ ho citato una nella mia nota 24 . 
Ma ignoranti di questo fatto, ben presto i sovrani ispanici decisero quale doveva essere il limite massimo delle eccessive usure che potevano percepire gli ebrei: nella Corona d'Aragona e nel regno di Navarra il limite fu stabilito nel $20 \%$, mentre che nella Corona di Castiglia fu fissato nel $33,3 \%$ (tranne un breve periodo in cui fu ridotto al $25 \%)^{27}$.

\subsubsection{Divieti di occupare cariche pubbliche}

La seconda decisione concerne il divieto di assumere cariche pubbliche, situazione che avrebbe significato superiorità dell'ebreo sul cristiano. Non sono stato capace di decidere perché questo canone fu ignorato nella stragrande maggioranza di nazioni. Il solo esempio che conosca è quello dei diversi stati integranti la Corona d'Aragona, dove a dicembre 1283 - gennaio 1284 furono dettate leggi definitive che letteralmente prescrivono

Item concedimus capitulum quod aliquis judeus non possit uti jurisdictionem vel districtu super christianos ${ }^{28}$.

\subsubsection{Obbligo di indossare vestiti e segni distintivi}

Se il IV Lateranense decidette la distinzione nell'abbigliamento, salta subito la domanda: perché vestiti diversi? per evitare qualsiasi possibilità di contatti? contatti fisici e/o morali? Sia quale sia la ragione, sta di fatto che la coesistenza c'era, ma però la convivenza veniva ostacolata. Ad ogni modo, ripetendo quanto ho detto spesso, l'obbligatorietà della distinzione può esser interpretata in due maniere: era un segno di obbrobrio o un segno distintivo, anche se esiste la via di mezzo, cioè, pensare che fosse obbrobrio-differenziante

\footnotetext{
27 Alcuni particolari sono riuniti in D. RomaNo, "Alfonso X y los judíos", § 4., ed anche in D. Romano, "Prestadores judíos», § 1. Cf. D. Romano, «Marco jurídico» (citato nella mia nota 15 ).

${ }^{28}$ D. Romano, Judíos al servicio de Pedro el Grande de Aragón (1276-1285), Barcelona 1983, pag. 177. Ho copiato il testo barcellonese; ma nelle pagine precedenti figurano quelli riguardanti i regni di Aragona e Valenza.
} 
o differenzio-obbrobriante. Ad ogni modo, però, era risultato di una necessità ${ }^{29}$.

\subsubsection{Separazione di habitat}

Per quanto poi riguarda la separazione di habitat, il fatto sta che fu una realtà sin dall'inizio della storia documentata degli ebrei ispanici. Ne parlai specificamente in un congresso francese ${ }^{30}$, descrivendo le singole forme di habitat (quartiere, via, isolatamente) ${ }^{31}$, quartieri e vie a se pur non formando un ghetto ${ }^{32}$.

A questo si aggiunssero poi, nel secondo decennio del ' 400 , le ordinanze dettate dalla regente $\mathrm{D}^{\mathrm{a}}$ Catalina per la Corona di Castiglia e ben presto applicate nella Corona d'Aragona e poi generalizzate dall'antipapa Benedetto XIII. Ma queste ordinanze sorgono da una nuova situazione concreta, dalla volontà di separare ebrei e convertiti.

Ho parlato altrove della mancanza di habitat rurale ${ }^{3 .}$.

\subsection{Diversità di costumi: due mogli contemporaneamente}

La diversità di costumi era conseguenza diretta della diversità di religione. Il tema è ancora ai prodromi pur se di recente sono stati fatti assaggi ma quasi tutti centrati sui costumi dei convertiti o dei processati dall'Inquisizione ${ }^{34}$.

${ }^{29}$ Le formulazioni più recenti si trovano in D. ROMANO, "Característiques dels jueus", § 1.1, poi tradotto e addattato nel $\S 1.1$ ( «Raza y religión») della relazione "Rasgos de la minoría judía en la Corona de Aragón", ed anche in "Judíos frente a cristianos en la Edad Media hispánica". Pensiamo che negli Stati Uniti di America a nessuno li è venuto in mente, almeno fino ad oggi, costringere un negro a portare un segno di questo genere.

${ }^{30}$ D. Romano, "Habitats urbains des juifs hispaniques", in Les sociétés urbaines en France méridionale et en Péninsule Ibérique au Moyen Age, Paris 1991, 421-434.

${ }^{31}$ Per le denominazioni degli habitat si veda D. Romano, "Aljama frente a judería, call y sus sinónimos", Sefarad XXXIX (1979) 347-354 [ristampato in D. Romano, De historia, 275-282]. E tengo ad accennare all'interessante notizia di A. Blasco MARTíneZ, “"Ebreismo”, sinónimo de judería", Sefarad XLI (1981) 111-113.

${ }^{32}$ No ho parlato nel $\S 1.5$ della mia relazione «Judios frente a cristianos en la Edad Media hispánica"

${ }^{33}$ Si veda la mia nota 23.

34 D. Romano, “Creencias y prácticas religiosas de los judíos en Valencia (14611492). Propuestas metodológicas a base de documentos inquisitoriales", in Luís de Santàngel $i$ el seu temps, Valencia 1992, 431-448, relazione al Congresso "Lluis de Santàngel i el seu temps" (Valencia 5-8 ottobre 1987). Si vedano anche gli articoli di Encarnación Marín, pubblicati nella rivista Sefarad, uno dei quali è stato citato nella mia nota 22. 
L'aspetto che merita un posto precipuo è il fatto che gli ebrei ispanici potevano avere due mogli contemporaneamente. Non parlo di bigamia giacché è parola che in italiano (ed in spagnolo) ha un senso peggiorativo ed abominevole.

Forse questo era l'aspetto più rilevante dei costumi sociali e materiali degli ebrei ispanici. È un uso con radici bibliche e perfino persistente ai giorni nostri, che nel Medioevo costituiva una peculiarità ispanoebrea, causa di rimprovero da parte degli ebrei aschenaziti, come già mise in rilievo R. Geršom di Magonza ${ }^{35}$. Ma nella Penisola Iberica la realtà era questa. Ammessa per consuetudine l'appartenenza dell'ebreo al sovrano, ciò lo costringeva a chiedere permesso per poter avere le due mogli, permesso che sempre veniva concesso -non conosco casi di negativa-, benché con una sfumatura: il concessionario era costretto a pagare una somma, piccola comunque, per il permesso, come appare scritto ai margini dei registri della cancelleria aragonese. Magari, forse per lapsus calami, perfino viene concessa l'autorizzazione per avere più di due mogli: questo è successo nel caso del celebre filosofo e uomo politico Hasdai Cresques ${ }^{36}$.

\subsection{La rottura della coesistenza e della tolleranza}

\subsubsection{Linee di forza}

All'interno della situazione di coesistenza, il gruppo maggioritario cristiano imponeva le sue leggi, norme se volete, alla minoranza ebrea, tollerata ma in un certo qual senso estromessa in forma di emarginazione.

Benché le notizie sussistenti suggeriscano che sovente le autorità cristiane ed i singoli individui rispettavano gli ebrei, la realtà rivela che una cosa è il rispetto e la tolleranza ed un altra ben diversa è la superazione totale delle differenze. Non si deve dimenticare che la religione di entrambe le comunità era completamente differente, e molti divari nascono dalla diversità di religione anche se talvolta questa sia soltanto una scusa che può celare cose molto diverse.

"ח Sull'argomento deve leggersi l'articolo di Y. Assis, כרס דרבינו גרשוס' ונישואי " כפל בספרד [The 'Ordinance of Rabbenu Gershom' and polygamous marriages in Spain] Zion XLVI (5741/1981) 251-277.

${ }^{36}$ F. BAER, Die Juden im christlichen Spanien. Urkunden und Regesten, vol. I, Berlin 1929, doc. $n^{2} 452$, pag. 711-712. 
La situazione di tolleranza con emarginazione o, viceversa, di emarginazione con tolleranza venne stabilita nel IV concilio lateranense (1215). Questa tolleranza fu rotta molte volte (sempre che era conveniente) sia da iniziativa popolare o pseudopopolare (voglio dire, aizzata) sia da iniziativa più o meno ufficiale della Chiesa o dello stato; se lo preferite: dal sovrano delicatamente spinto dalla Chiesa.

Talvolta, indubbiamente troppo spesso dalla prospettiva degli ebrei, la rottura della tolleranza potè esprimersi in diversi modi, tra i quali sono da rilevare gli sforzi per convincere gli ebrei a trasferirsi al cristianesimo. Erano costretti a sopportare tentative di conversione al cristianesimo che potevano manifestarsi in due maniere: $1^{a}$ ) sotto la forma pacifica di predicazioni e di quelli che io chiamo addottrinamenti o $2^{\mathrm{a}}$ ) sotto forma di brutali attacchi come succedette in occasione della grande ondata persecutoria del 1391, funesta per l'ebraismo ispanico e ben probabilmente originata da motivi economicosociali piuttosto che religiosi.

\subsubsection{I principali fatti cronologicamente disposti}

La prima rottura grave della coesistenza ebbe luogo nel 1391. Dopo i moti di quell'anno e malgrado la riduzione demografica, il numero degli ebrei che perseverarono nella loro fede fu relativamente grande: questo spiega che si pensasse ad altri mezzi, che sarebbero stati cagione di nuove rotture.

La seconda rottura fu marcata dalle ordinanze di D. ${ }^{a}$ Catalina (1412), che decidette la chiusura delle giudecche per evitare i contatti tra ebrei e cristiani nuovi in modo da evitare un possibile influsso degli ebrei sui testè convertiti ${ }^{37}$. Quasi immediatamente (1414) furono applicate nella Corona d'Aragona da suo cognato Ferdinando I di Antequera, ed in seguito raccolte e generalizzate in una bolla dell'antipapa Benedetto XIII (11 aprile 1415), dettata dopo l'addottrinamento di Tortosa.

La terza rottura ebbe le sue origini dal fiasco della conversione alla quale forse miravano i moti antiebrei del 1391 e dall'incertezza del successo delle misure di separazione dei due gruppi (ordinanze

\footnotetext{
${ }^{37} \mathrm{Ne}$ ho parlato altrove, nel $\S 1.2 .4 .1$ della mia relazione «Judíos hispánicos», dove copiai un breve frammento delle ordinanze.
} 
di D. ${ }^{a}$ Catalina). Allora l'assorbente antipapa Benedetto XIII suggerì di organizzare un incontro che aveva come scopo la conversione pacifica degli ebrei: è l'addottrinamento di Tortosa, di cui parlerò oltre $(\S 3.3 .2 .2)$.

Da allora in poi e durante tutto il ' 400 il problema religioso si aguzzò perché in una stessa famiglia alcuni individui restarono fideli alla loro religione mentre alcuni altri si convertivano, più o meno sinceramente. Più tardi, alla fine del ' 400 si sommerà a questo la nascita dell'Inquisizione moderna - non la medioevale, che in certo modo era qualcosa di ben diverso. Tra i punti che bisogna osservare vale la pena ricordare la diffidenza degli ebrei ed il timore dei convertiti.

Malgrado le misure previste non fu possibile eliminare il problema dei convertiti, che avrebbe dovuto sopravvivere perfino dopo l'espulsione del 1492 (o degli anni successivi ${ }^{38}$ ).

\subsubsection{Le rotture mentali}

Accanto a queste misure a carattere fisico, si schierano quelle che ben possono venir considerate rotture mentali, e che non penso che sia troppo osato definirle come forme di cattiveria. Una prima classificazione permeterebbe distinguere due grandi tipi di azione: le prediche obbligatorie e gli addottrinamenti, anch'essi non volontari.

\subsubsection{Le prediche cristiane ${ }^{39}$}

Pur non essendo un tema studiato, si conoscono parecchie indicazioni sulle prediche cristiane agli ebrei. Queste prediche sono attestate in tutte le nazioni ispaniche, benché non lo siano tanto quanto nella Corona d'Aragona, dove ci sono note grazie ad un saggio parziale che prende come bersaglio soltanto il ' 200 ed il ' 300 , cioè, due secoli ${ }^{40}$. Di esse la prova più spesso citata e magari

${ }^{38} 1493$ nel Rossiglione; 1496-1497 nel Portogallo; 1498 nel regno di Navarra.

${ }^{39}$ Seguo da vicino le mie parole, esposte in D. ROMANO, “Llull e la cultura ebraica. Tentativo di sistematica", Annali. Sezione Romanza XXXIV (= Atti del Convegno Internazionale Ramon Llull, il lullismo internazionale, l'Italia [Napoli 30 e 31 marzo, 1 aprile 1989]) (1992) 171-189.

${ }^{40}$ Vedi J. RIERA I SANS, "Les llicències reials per predicar als jueus $\mathrm{i}$ als sarraïns (segles XIII-XIV)", Calls 2 (1987) 113-143. 
riprodotta ${ }^{41}$ contiene l'autorizzazione di Giacomo II (1299) affinché il maestro Ramon Llull possa esporre la verità della fede cattolica agli ebrei (ed anche ai musulmani). L'autorizzazione indica che gli ebrei saranno costretti ad ascoltare il sermone ed avranno la possibilità, ma non l'obbligo, di rispondere. Il permesso contiene elementi proprio negativi quanto all'atteggiamento ispanico riguardo agli ebrei: la predica si terrà nelle sinagoghe il sabato e la domenica, cioè, si direbbe che c'è una vulnerazione del rispetto e della tolleranza e quasi oserei dire con obbrobrio e malignità mentale: in sabato e nella sinagoga!

\subsubsection{Gli addottrinamenti cristiani}

I tentativi di assimilazione cristiana in forma più pubblicitaria si sogliono chiamar «dispute». Ma da parecchio tempo ho proposto e costantemente propongo di sostituire la parola "disputa» per quella di "addottrinamento». Mi spiego. Gli incontri erano organizzati in tal modo che non c'era nessuna possibilità di sconfitta cristiana. A quei tempi - forse lo stesso dovrebbe dirsi di qualsiasi altro- una polemica bandita da una delle parti e tenuta in terreno non neutrale, in fondo era inutile e con risultato prevedibile, inutile tranne a scopi propagandistici. Per ciò tengo a patrocinare un cambiamento di denominazione.

Tranne esempi parigini ${ }^{42}$, gli addottrinamenti si conoscono nella Corona d'Aragona. A quanto pare il primo ebbe luogo a Barcellona nel 1263 in presenza del re Giacomo I. Conta con una copiosa bibliografia, basata a seconda dei casi sulle fonti ebraiche ed anche sugli atti cristiani, nei quali figura questa frase:

non ut fides Domini Jhesu Christi, quae propter sui certitudinem non est in disputatione ponenda..., sed ut ipsius fidei veritas manifesteretur propter destruendos Judeorum errores et ad tollendam

${ }^{41}$ Il documento fu pubblicato da A. RuBió I Lluch, Documents per a la història de la cultura catalana mig-eval, vol. I, Barcelona 1908, pag. 13-14, doc. XIV. Bisogna rilevare che non si sa null'altro di concreto sull'attività predicatrice di Llull.

${ }^{42}$ Oltre a quello del 1240 , si veda J. Shatzmiller, La deuxième controverse de Paris. Un chapitre dans la polémique entre chrétiens et juifs au Moyen Age, Paris Louvain 1994. 
confidentiam multorum Judeorum, qui cum non possent suos errores deffendere ${ }^{43}$.

Secolo e mezzo più tardi, sempre nella Corona d'Aragona, regnante re Ferdinando I ma dietro suggerimento di Benedetto XIII, ebbe luogo l'addottrinamento di Tortosa (1413-1414), che la professoressa Asunción Blasco (dell'Università di Saragozza) ${ }^{44}$ pensa giustamente che fu conseguenza abbastanza diretta del fiasco di quanto si pensò ottenere con i moti del 1391 ed anche con le ordinanze del 1412.

A somiglianza del caso di Barcellona, la caratteristica più saliente dell'addottrinamento di Tortosa era la volontà di dimostrare la verità cristiana tramite testi del Talmud. Le fonti ebraiche e latine coincidono quasi parola a parola, come si può accertare dalla lettura di alcuni frammenti.

Un anonimo manoscritto ebraico, ormai sparito, spiega che

Il suo proposito [del Papa] quando convocò questa controversia non era quello di discutere sulla religione degli ebrei e dei cristiani, quale delle due fosse vera, perché era ben conscio che la religione dei cristiani era la vera, tanto ai suoi occhi quanto a quelli di qualsiasi cristiano: la sua intenzione era far vedere e dimostrare agli ebrei che nel Talmud è ben chiaro che il Messia è già arrivato ${ }^{45}$.

Gli atti cristiani, in latino, scritti dopo la fine dell'addottrinamento, nel rendiconto dell'ottava seduta inseriscono questa frase dell'antipapa:

intentionem domini nostri principaliter, non ad disputandum sed ad

${ }^{43}$ E. C. Girbal, Los judíos en Gerona, Gerona 1870, pag. 66 [ristampato in D. Romano (ed.), Per a una història de la Girona jueva, Girona 1988, vol. I, pag. 92].

44 A. BlasCo, "El impacto de los ataques de 1391 y del adoctrinamiento de Tortosa en la sociedad judía aragonesa", relazione alle III Jornadas hispanoportuguesas de Historia Medieval, "La Península Ibérica en la Era de los descubrimientos, 1391-1492", sezione "El final de la convivencia: judíos y conversos en la Península Ibérica al final de la Edad Media» (Sevilla 25-29.11.1991), tuttora inedita.

45 Traduco dalla versione catalana pubblicata da J. Riera I SANS, La Crònica en hebreu de la Disputa de Tortosa, Barcelona 1974, pag. 18. 
dictos iudeos in fide catholica convertendum (che una variante sostituisce per «informandum») ${ }^{46}$.

Alla fine del '400 la cronaca ebraica denominata Šebet Yěhûdâ ripete quasi letteralmente le frasi dell'anonimo sopraccitato nel dire

Savi ebrei! Dovete sapere che non sono venuto né vi ho riuniti per dimostrare quale delle due religioni sia la vera, che ben so che la mia religione e la mia fede è la vera e che la vostra Legge era vera ma fu derogata. Non siete venuti se non perché Girolamo vuol dimostrare tramite il Talmud dei vostri rabbini (che ne sapevano di più di voi) che il Messia è gia venuto. Non parlate, dunque, davanti a me null'altro che di questo tema ${ }^{47}$.

\section{Conclusione}

Per concludere, io direi che dal punto di vista cristiano la tolleranza esisteva, senza sospetto di dubbio sotto la sua forma teorica, ma anche nella realtà. E lo stesso potrebbe dirsi dell'emarginazione. Ma è evidente che l'ebreo non sposa una cristiana né il cristiano una ebrea; inoltre, si cerca di ridurre i contatti alla sfera sociale o alla sfera economica, alla sfera della coesistenza più o meno pacifica. Insisto sulla mia affermazione: coesistenza non vuol mica dire convivenza giacchè c'era allora, ed in grandissima parte persistono oggi, una serie di topici antiebraici, che richiedono stentoreamente la soppressione definitiva.

${ }^{47}$ Utilizzo l'edizione ebraica di I. BAER, Yerusalaim 5707/1946-1947, § 40, pag. 96. 


\section{RESUMEN}

Se intenta debatir y aclarar el dilema entre "coexistencia" y "convivencia", dos palabras que, contra lo que se dice y cree, no son sinónimas. Se defiende la tesis de que entre cristianos e hispanojudíos hubo coexistencia pero no convivencia (y menos si se piensa en el francés "cohabitation"), visto desde el punto de vista de la terna filosofia, teoría y, sobre todo, realidad; pensando siempre en el respeto, tolerancia y marginación de una minoría por parte de una mayoría dominante.

\section{SUMMARY}

In this article an attempt is made to discuss and clarify the dilemma between "coexistence" and "living together", which - against what it is often said or believedare not synonimous. The statement that Christians and Spanish-Jews coexisted but did not live together (especially if this concept is considered as in French "cohabitation") is seen from the philosophical, theoretical, and, in particular, realistic point of view; having always into account the respect, tolerance, and margination of a minority by a dominant majority. 